\title{
Predictive values profiling of interleukin-2, interleukin-8, tumor necrosis factor- $\alpha$, procalcitonin, and C-reactive protein in critical gastrointestinal cancer patients
}

\author{
Yamin Yan ${ }^{1}$, Zhenghong Yu ${ }^{1}$, Jingjing $\mathrm{Lu}^{1}$, Peili Jin ${ }^{1}$, Zhaoqing Tang ${ }^{2}$, Yan $\mathrm{Hu}^{1}$ \\ ${ }^{1}$ Nursing Department, Zhongshan Hospital, Fudan University, Shanghai, China; ${ }^{2}$ General Surgery Department, Zhongshan Hospital, Fudan \\ University, Shanghai, China \\ Contributions: (I) Conception and design: Y Hu; (II) Administrative support: None; (III) Provision of study materials or patients: Z Tang; (IV) \\ Collection and assembly of data: Y Yan, Z Yu, J Lu, P Jin; (V) Data analysis and interpretation: All authors; (VI) Manuscript writing: All authors; (VII) \\ Final approval of manuscript: All authors. \\ Correspondence to: Yan Hu. Head Nurse of the Surgery Department (Ward 10), Zhongshan Hospital, Fudan University, 180, Fenglin Road, Xuhui \\ District, Shanghai 200032, China. Email: hu.yan@zs-hospital.sh.cn; Zhaoqing Tang. General Surgery Department, Zhongshan Hospital, Fudan \\ University, 180, Fenglin Road, Xuhui District, Shanghai 200032, China. Email: tang.zhaoqing@zs-hospital.sh.cn.
}

\begin{abstract}
Background: The prognostic values of serum cytokines in cancer have not yet been fully determined. The objective of this study was to identify potential biomarkers associated with clinical outcomes in critical gastrointestinal (GI) cancer patients.

Methods: A retrospective analysis was performed to quantify serum interleukin (IL)-2, IL-8, tumor necrosis factor- $\alpha$ (TNF- $\alpha$ ), procalcitonin (PCT), and C-reactive protein (CRP) for correlation with clinical outcomes in GI cancer patients. The patients were divided into tertiles or quartiles based on the cytokine levels: Q1, Q2, and Q3, or Q1, Q2, Q3, and Q4. Receiver operating characteristic (ROC) curves were drawn to determine the optimal cutoff values of the cytokines.

Results: Trend analysis showed that IL-2, IL-8, TNF- $\alpha$, PCT, and CRP levels had significant positive correlations with mortality in GI cancer patients (all P-values were lower than 0.05 ). The significance was observed in Q3 vs. Q1 in IL-2 (P=0.026), Q3 vs. Q1 in IL-8 (P=0.003), Q2 and Q3 vs. Q1 in TNF- $\alpha(\mathrm{P}=0.012$ and $\mathrm{P}=0.002$, respectively), $\mathrm{Q} 4$ vs. $\mathrm{Q} 1$ in $\mathrm{PCT}(\mathrm{P}=0.031), \mathrm{Q} 3$ and $\mathrm{Q} 4$ vs. $\mathrm{Q} 1$ in $\mathrm{CRP}(\mathrm{P}=0.011$ and $\mathrm{P}=0.001$, respectively). The area under curve (AUC) of IL-2, IL-8, TNF- $\alpha$, PCT, and CRP were 0.706, 0.729, 0.743, 0.769 , and 0.736 , and the optimal cutoff points were determined at $838 \mathrm{U} / \mathrm{mL}, 46.15 \mathrm{pg} / \mathrm{mL}, 11.95 \mathrm{pg} / \mathrm{mL}$, $0.77 \mathrm{pg} / \mathrm{mL}$, and $109.38 \mathrm{mg} / \mathrm{L}$, respectively. Under these critical values, the sensitivity was $73.3 \%, 66.7 \%$, $80.0 \%, 93.3 \%$, and $86.7 \%$, and the specificity was $64.9 \%, 72.0 \%, 60.4 \%, 61.8 \%$, and $68.9 \%$, respectively.
\end{abstract}

Conclusions: In GI cancer patients, serum IL-2, IL-8, TNF- $\alpha$, PCT, and CRP levels can provide potential prognostic values for predicting clinical outcomes. The results may facilitate the exploration of cancerrelated cytokine networks and development of novel therapy for GI cancer patients.

Keywords: Cytokines; gastrointestinal cancer (GI cancer); prognostic value

Submitted May 25, 2021. Accepted for publication Jul 06, 2021.

doi: 10.21037/jgo-21-334

View this article at: https://dx.doi.org/10.21037/jgo-21-334

\section{Introduction}

Gastrointestinal (GI) cancer poses a high-threat risk to public health, and it accounts for approximately one-third of the total global cancer incidence and mortality (1). In the USA, GI cancers are a societal burden with 319,160 new cases and 160,820 GI cancer-related deaths reported in 2018 (2). Prognostic assessment for cancer patients has remained challenging, and the use of a novel biomarker 
test in scientific research and clinical practice may provide insight for personalized treatments and result in better outcomes $(3,4)$.

Inflammatory responses play pivotal roles in the development of cancer, and are the major cause of morbidity and mortality in cancer patients. Considerable attention has been given to the potential value of serologic biomarkers, such as pro- and anti-inflammatory cytokines, in early stage identification of high-risk patients. Cytokines are small molecule proteins synthesized and secreted by immune cells (such as monocytes, macrophages, T cells, B cells, NK cells, etc.) and some non-immune cells (endothelial cells, epidermal cells, fibroblasts, etc.). The biological function is regulating innate and adaptive immunity, and involved in biological processes via binding to specific receptors $(5,6)$. As multifunctional molecules, these inflammatory biomarkers not only damage cancer cells but also act as important mediators in the killing of cancer cells (7). Increasing studies have demonstrated that defects in immune function, especially cellular immune dysfunction, occur commonly in cancer patients, and cancer-related outcomes are always related to higher levels of circulating cytokines (8). More recently, cytokines have been recognized as important mediators linking inflammation and cancer, and potential therapeutic and preventive targets as well as prognostic factors (9). For example, in colorectal cancer (CRC), high serum interleukin-6 (IL-6) level correlates with advanced pathological tumor stage and metastatic disease (10). Some researchers built a diagnostic model for gastric cancer (GC), and found that IL- 6 , IL- 8 , and TNF- $\alpha$ may potentially serve as a screening method for the early detection of GC (7). Higher levels of pro-inflammatory cytokines are linked to cancer-related outcomes. In breast, lung, and prostate cancer patients, the cytokines [granulocytemacrophage colony-stimulating factor (GM-CSF), interferon- $\gamma$ (IFN- $\gamma$ ), IL-1 $\beta$, IL-2, IL-4, IL-5, IL-12p70, IL-17A, and IL-23] are found negatively correlated with pain scores, and can be used to predict pain severity (11).

Previous studies have confirmed the correlation of immune dysfunction and cancer, including cancer initiation, promotion, progression, and metastasis, and cytokines' level may vary with cancer stage, subtype, chemotherapy/ treatment, and so on (12). Therefore, it is difficult to demonstrate the association of clinical outcome or cancerrelated symptom with any single specific biomarker. Although prognostic scores based on systemic inflammation markers such as C-reactive protein (CRP) and neutrophil count have been studied (13-15), predicting morbidity and mortality in cancer patients remains challenging, and there is ongoing controversy regarding the extent of what cytokines' level can inform the prediction of cancer patient outcomes.

In an effort to explore prognostic values of cytokines in GI cancer and generate new data for formulating more individualized immune therapy regimens for GI cancer patients, we detected the circulating level of IL-2, IL-8, tumor necrosis factor- $\alpha(\mathrm{TNF}-\alpha)$, procalcitonin (PCT), and CRP, and investigated the sensitivity and specificity in predicting mortality in critical GI cancers. The findings may provide a new direction for exploring the pathological mechanism and also provide a potential means for treating and predicting clinical outcomes in GI cancer patients. We present the following article in accordance with the REMARK reporting checklist (available at https://dx.doi. org/10.21037/jgo-21-334).

\section{Methods}

\section{Setting and patients}

This study was conducted in a large teaching hospital in China (Clinical Trials Identifier: ChiCTR2000033894).

From 1 January 2018 to 31 December 2020, a total of consecutive 408 patients with critical GI cancer who were transferred to the surgical intensive care unit (SICU) after surgery for further treatment were recruited to this study. All patients received rule-based perioperative, anesthesia management, and critical care. The inclusion criteria were: (I) age $\geq 18$ years old; (II) patients received elective or urgent major surgery therapy; and (III) written informed consent was provided. The exclusion criteria were: (I) a history of systemic inflammatory disease; (II) treated with glucocorticoid or cytokine/anti-cytokine therapy within the previous year; (III) presence of severe bacterial infection or sepsis; or (IV) baseline data (serum cytokine levels, clinical characteristics) were missing within $24 \mathrm{~h}$ after SICU admission.

This study was carried out retrospectively. Before the analysis, the information of participants was anonymized and deidentified. All procedures performed in this study involving human participants were in accordance with the Declaration of Helsinki (as revised in 2013), and approval to conduct this study was granted by the Ethics Committee on Human Experimentation of Zhongshan Hospital, Fudan 
University (B2020-107R).

\section{Criteria for critical GI cancer}

The criteria were established by the general surgery department of Zhongshan Hospital, Fudan University. If 1 of the following was satisfied, it was considered critical GI cancer, including:

(I) Age $\geq 85$ years.

(II) Severe dysfunction of any vital organ:

(i) Severe pulmonary function dysfunction.

(ii) Heart insufficiency/heart failure [New York Heart Association (NYHA) grade II or above].

(iii) Patients with uremia requiring abdominal dialysis or hemodialysis maintenance.

(iv) Liver dysfunction (Grade Child B or C); liver cirrhosis; portal hypertension.

(v) Immunosuppression state: long-term use of hormones, immunosuppressants, cytotoxic drugs, and so on.

(vi) Diabetic patients: poor blood glucose control; with diabetes complications.

(III) Insufficiency or pathological changes in 2 or more vital organs.

(IV) Acute cardiovascular and cerebrovascular disease having occurred within 6 months, such as myocardial infarction, cerebral infarction, cerebral hemorrhage, and so on.

(V) Intraoperative combined organ resection (except cholecystectomy).

(VI) Postoperative unplanned reoperation.

(VII) Other patients identified with difficult or critical diseases after discussion of a multidisciplinary team.

\section{Cytokine measurements}

Blood specimens for cytokines were collected between 6:00 and 7:00 am in the first $24 \mathrm{~h}$ after SICU admission. The blood was centrifuged for 10-15 min at 3,000 rpm, and the serum was stored at $-80^{\circ} \mathrm{C}$ until it was assayed. According to the manufacturer's instructions, an IMMULITE 1000 Immunoassay System (Siemens Healthcare Diagnostics $\mathrm{GmbH}$, Berlin, Germany) was used to detect the serum concentrations of IL-2, IL- 8 , TNF- $\alpha$, and PCT. The inter- assay precision of IL-2, IL-8, TNF- $\alpha$, and procalcitonin were in the range $2.9-3.7 \%, 3.6-3.8 \%, 2.6-3.6 \%$, and $1.4-6.9 \%$, respectively; and the intra-assay precision were in the range $6.1-8.1 \%, 5.2-7.4 \%, 4.0-6.5 \%$, and $1.5-7.9 \%$, respectively. The normal ranges were 223 to $710 \mathrm{U} / \mathrm{mL}$ for IL-2, $<62 \mathrm{pg} / \mathrm{mL}$ for IL- $8,<8.1 \mathrm{pg} / \mathrm{mL}$ for TNF- $\alpha$, $<0.5 \mathrm{ng} / \mathrm{mL}$ for PCT, and $0-3 \mathrm{mg} / \mathrm{L}$ for CRP. The tests were conducted by trained medical technicians who were blinded to the clinical data.

\section{Data collection}

The primary outcome was hospital mortality. The following data were collected within $24 \mathrm{~h}$ after SICU admission: demographic information, including age and gender; admission diagnosis; cytokine levels, type of organ support [e.g., mechanical ventilation or continuous renal replacement therapy (CRRT)]. Data were collected by trained collectors from admission to death or discharge from the SICU.

\section{Statistical analysis}

Categorical variables were presented as numbers (percentages), and continuous variables with normal distribution were presented as mean \pm standard deviation (SD), non-normal variables were reported as median (interquartile range). In case of missing data, average value was used to interpolate the missing value.

Cytokine levels were classified into tertiles or quartiles based on the distribution: Q1 (which also included untested cases), Q2, and Q3, or Q1, Q2, Q3, and Q4. Chisquare was used to analyze differences between groups for categorical variables, and $t$-test or Mann-Whitney $\mathrm{U}$ test for continuous variables. The odds ratio (OR) and $95 \%$ confidence intervals (CI) were calculated. Using Q1 as a reference, we assessed ORs for the Q2, Q3, and Q4. According to the results of the univariate analysis, multivariate regression analyses were performed to identify independent risk factors for mortality after adjustment for confounding variables. Ascending tertiles or quartiles of cytokine levels were treated as ordered categories when conducting trend analysis. Receiver operating characteristic (ROC) curves were established for the optimal cutoff values, sensitivity, and specificity of IL-2, IL-8, TNF- $\alpha$, PCT, and CRP level. 
Table 1 Participant clinical characteristics

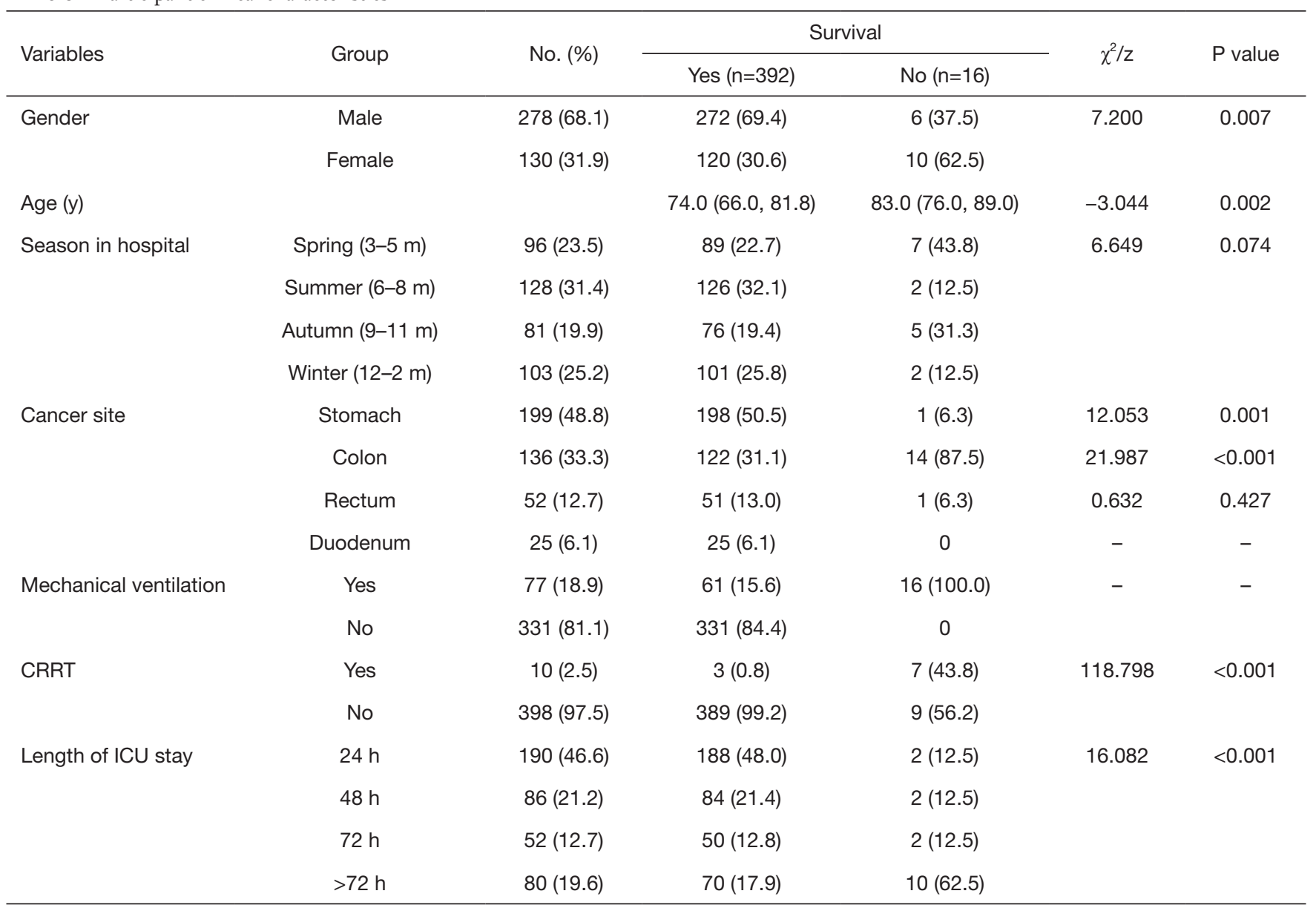

CRRT, continuous renal replacement therapy; ICU, intensive care unit.

Statistical analysis was performed using the software SPSS 26.0 (SPSS Inc., IBM Corp., Chicago, IL, USA). A P value $<0.05$ indicated statistical significance.

\section{Results}

\section{Clinical characteristics}

As shown in Table 1, a total of 408 critical GI cancer patients were included, among whom 392 cases survived and 16 died, with a mortality rate of $3.9 \%$. Non-survivors had a significantly older age compared with survivors, with a mean age of 83.0 years $(\mathrm{P}=0.002)$. The non-survivor group also had higher rates of female patients, colon cancer patients, mechanical ventilation and CRRT therapy. When the length of ICU stay $>72 \mathrm{~h}$, the proportion of non- survivors was significantly higher $(\mathrm{P}<0.001)$. Groups did not differ significantly by season in hospital $(\mathrm{P}=0.074)$.

\section{Cytokine levels in survivors and non-survivors}

Table 2 summarizes the association of each cytokines' level and clinical outcome. Serum IL-2, IL-8, TNF- $\alpha$, PCT, and CRP levels in non-survivors were significantly higher than those in the survivors. The levels of IL-2, IL-8, and TNF- $\alpha$ were divided into tertiles: Q1 (which included untested cases), Q2, and Q3. The proportion of nonsurviving patients in the highest tertile $(\mathrm{Q} 3)$ were all higher than those in the lowest tertile (Q1). The levels of PCT and CRP were classified into quartiles: Q1 (which also included untested cases), Q2, Q3, and Q4. The proportion of nonsurviving patients in the high quartiles (Q3 and Q4) were all 
Table 2 Cytokine levels in survivors and non-survivors

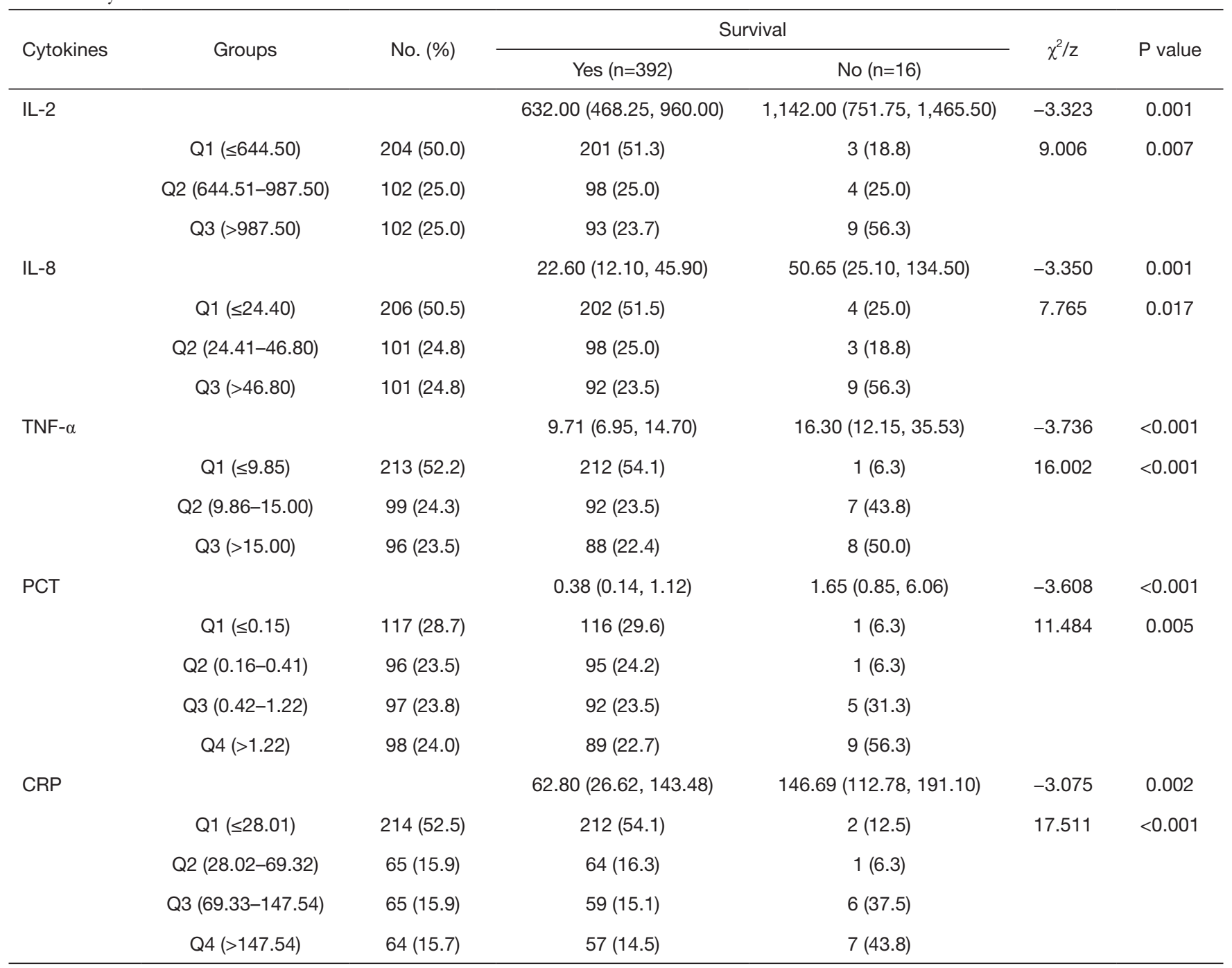

IL-2, interleukin-2; IL-8, interleukin-8; TNF- $\alpha$, tumor necrosis factor- $\alpha$; PCT, procalcitonin; CRP, C-reactive protein.

higher than those in the lowest quartile (Q1).

\section{Trend analysis of each cytokines' tertiles or quartiles}

IL-2, IL-8, TNF- $\alpha$, PCT, and CRP levels were fitted with logistic regression analysis in the form of grade variables. The results showed that all the cytokines' levels had significant linear correlations with patient outcomes, all the $\mathrm{P}$-value for trend analysis were $<0.05$. The levels of IL-2 (OR $=4.951,95 \%$ CI: 1.206 to 20.319$)$ and IL-8 $(\mathrm{OR}=6.973$, 95\% CI: 1.895 to 25.663 ) in Q3 were significantly higher than those in the lowest tertile (Q1). The levels of TNF- $\alpha$ in $\mathrm{Q} 2(\mathrm{OR}=16.027,95 \% \mathrm{CI}: 1.861$ to 138.002 ) and Q3 ( $\mathrm{OR}=30.856,95 \%$ CI: 3.530 to 269.683 ) were significantly higher than that in Q1. For PCT, the levels in Q4 (OR $=10.463,95 \%$ CI: 1.238 to 88.442 ) were significantly higher than that in the lowest quartile (Q1). The levels of CRP in $\mathrm{Q} 3(\mathrm{OR}=9.115,95 \% \mathrm{CI}: 1.643$ to 50.570$)$ and $\mathrm{Q} 4(\mathrm{OR}$ $=19.441,95 \%$ CI: 3.430 to 110.197$)$ were significantly higher than that in Q1 (Table 3).

\section{Predictive value of IL-2, IL-8, TNF- $\alpha, P C T$, and CRP}

Table 4 demonstrates that the area under curve (AUC) of IL-2, IL-8, TNF- $\alpha$, PCT, and CRP were 0.706, 0.729, $0.743,0.769$, and 0.736 , respectively. This result indicated that these variables carry diagnostic significance for critical GI patients' clinical outcomes. The optimal cutoff values 
Table 3 Logistic regression analysis of each cytokines' tertiles or quartiles on patient outcomes

\begin{tabular}{|c|c|c|c|c|c|}
\hline Variable & \multicolumn{4}{|c|}{ OR $(95 \% \mathrm{Cl})$} & $P$ value for trend \\
\hline Model I: IL-2 & 1.000 & $2.220(0.444-11.105)$ & 4.951 (1.206-20.319) & & 0.022 \\
\hline$P$ values & & 0.332 & 0.026 & & \\
\hline Model II: IL-8 & 1.000 & $2.593(0.514-13.088)$ & $6.973(1.895-25.663)$ & & 0.003 \\
\hline Model III: TNF-a & 1.000 & $16.027(1.861-138.002)$ & 30.856 (3.530-269.683) & & $<0.001$ \\
\hline$P$ values & & 0.012 & 0.002 & & \\
\hline Model IV: PCT & 1.000 & $1.386(0.082-23.510)$ & $4.757(0.520-43.539)$ & $10.463(1.238-88.442)$ & 0.007 \\
\hline$P$ values & & 0.821 & 0.167 & 0.031 & \\
\hline
\end{tabular}

All models were adjusted for gender, age, whether gastric cancer, and whether colon cancer. OR, odds ratio; Cl, confidence interval; IL-2, interleukin-2; IL-8, interleukin-8; TNF- $\alpha$, tumor necrosis factor- $\alpha$; PCT, procalcitonin; CRP, C-reactive protein.

Table 4 Predictive values of IL-2, IL-8, TNF-a, PCT, and CRP

\begin{tabular}{lccccc}
\hline Parameters & IL-2 & IL-8 & TNF- $\alpha$ & PCT & CRP \\
\hline AUC & $0.706(0.583-0.829)$ & $0.729(0.618-0.840)$ & $0.743(0.637-0.848)$ & $0.769(0.683-0.856)$ & $0.736(0.623-0.849)$ \\
Cutoff value & $838 \mathrm{U} / \mathrm{mL}$ & $46.15 \mathrm{pg} / \mathrm{mL}$ & $11.95 \mathrm{pg} / \mathrm{mL}$ & $0.77 \mathrm{pg} / \mathrm{mL}$ & $109.38 \mathrm{mg} / \mathrm{L}$ \\
Sensitivity & $73.3 \%$ & $66.7 \%$ & $80.0 \%$ & $93.3 \%$ & $86.7 \%$ \\
Specificity & $64.9 \%$ & $72.0 \%$ & $60.4 \%$ & $61.8 \%$ & 2.44 \\
+LR & 2.09 & 2.38 & 2.02 & $0.9 \%$ & 2.79 \\
-LR & 0.41 & 0.46 & 0.33 & 0.11 & 0.19 \\
\hline
\end{tabular}

IL-2, interleukin-2; IL-8, interleukin-8; TNF- $\alpha$, tumor necrosis factor- $\alpha$; PCT, procalcitonin; CRP, C-reactive protein; AUC, area under the curve; LR, likelihood ratio.

for IL-2, IL-8, TNF- $\alpha$, PCT, and CRP, as determined by the maximum Youden index, were $838 \mathrm{U} / \mathrm{mL}, 46.15 \mathrm{pg} / \mathrm{mL}$, $11.95 \mathrm{pg} / \mathrm{mL}, 0.77 \mathrm{pg} / \mathrm{mL}$, and $109.38 \mathrm{mg} / \mathrm{L}$, respectively. Under these critical values, the sensitivity was $73.3 \%$, $66.7 \%, 80.0 \%, 93.3 \%$, and $86.7 \%$, and the specificity was $64.9 \%, 72.0 \%, 60.4 \%, 61.8 \%$, and $68.9 \%$, respectively. The positive likelihood ratios for IL-2, IL-8, TNF- $\alpha$, PCT, and CRP were 2.09, 2.38, 2.02, 2.44, and 2.79, and the corresponding negative likelihood ratios were $0.41,0.46$, $0.33,0.11$, and 0.19 , respectively (Table 4 and Figure 1).

\section{Discussion}

Cytokine expression analysis may be a promising method for predicting clinical outcome and provide a measurement to more objectively understand the complex nature of cytokine regulation and association with complications in cancer patients. A recent study by Qeadan et al. (16) explored map kinase-activated protein kinase 2 (MK2) and a novel associated cytokine panel in GC using a novel intercorrelation analysis approach. An interesting finding was that the MK2 pathway is strongly linked with metastasis and a panel of cytokines, including G-CSF, GMCSF, Mip-1 $\beta$, IFN- $\alpha$, MCP-1, IL-1 $\beta$, IL-6, and TNF- $\alpha$. Chen et al. (17) incorporated 19 cytokines that correlated with neutrophil-lymphocyte ratio into a cytokine score, and made a comparison between the composite score and individual cytokines, demonstrating an enhanced prognostic 

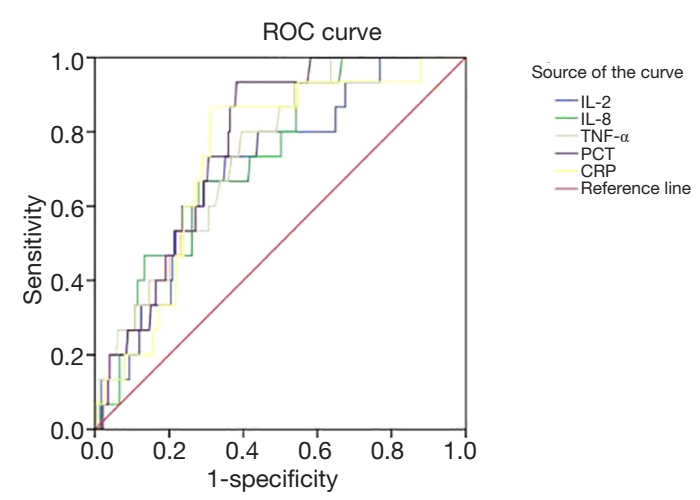

Diagonal segmentare produced by ties

Figure 1 Prognostic value of IL-2, IL-8, TNF- $\alpha$, PCT, and CRP for predicting clinical outcomes in critical GI cancer patients. IL-2, interleukin-2; IL-8, interleukin-8; TNF- $\alpha$, tumor necrosis factor- $\alpha$; PCT, procalcitonin; CRP, C-reactive protein; ROC, receiver operating characteristic.

ability in CRC. These studies show the potential clinical value of cytokines in predicting overall survival of GI cancer patients.

The current study demonstrates a prognostic analysis using serum cytokine expression level to predict clinical outcome in critical GI cancer patients. On the first day after SICU admission, the levels of IL-2, IL-8, TNF- $\alpha$, PCT, and CRP were significantly elevated in non-survivors, providing a potential value of cytokines that can predict overall survival. Our current study found a correlation between increased levels of IL-2, IL-8, TNF- $\alpha$, PCT, CRP and mortality [Q3 vs. Q1 in IL-2 $(\mathrm{P}=0.026), \mathrm{Q} 3$ vs. Q1 in IL-8 ( $\mathrm{P}=0.003), \mathrm{Q} 2$ and $\mathrm{Q} 3$ vs. $\mathrm{Q} 1$ in TNF- $\alpha(\mathrm{P}=0.012$ and $\mathrm{P}=0.002$, respectively), $\mathrm{Q} 4$ vs. $\mathrm{Q} 1$ in $\mathrm{PCT}(\mathrm{P}=0.031)$, $\mathrm{Q} 3$ and $\mathrm{Q} 4$ vs. $\mathrm{Q} 1$ in $\mathrm{CRP}(\mathrm{P}=0.011$ and $\mathrm{P}=0.001)]$. The trend analysis demonstrated significant differences of all the variables (all $\mathrm{P}$ values were lower than 0.05 ). Although previous study showed cytokines present different expression pattern across cancer types (18), prospective studies of GI cancer outcome will benefit from this particular study.

The precise prediction of prognosis in cancer patients is a challenging issue. Variability in the clinical and pathological characteristics of different cancers hinders simple stratification of patients into meaningful prognostic cohorts (19). Novel molecular tests provide insights for improving accurate prognostic assessments for cancer patients $(3,20)$. As hallmarks of systemic inflammation in cancer patients, cytokines have gained much interest in clinical practice. During the past decade, increasing findings have demonstrated that serum cytokine concentrations provide prognostic information in cancer patients. The levels of IL-6 are associated with advanced stage in CRC and GC, and higher IL-6 concentration is an independent predictive marker of poor prognosis and survival (21-23). Birgisson et al. (24) analyzed 92 cancer-related proteins in a cohort of stage II-IV CRC patients, including cytokines, chemokines, and growth factors. The results showed that CSF1, CXCL10, CXCL9, HGF, IL-6, osteoprotegerin, and PGF, were significantly associated with survival, and osteoprotegerin was the best in predicting survival in multivariable survival models. Liang et al. (25) determined the association of cytokine and stages in GC, and found that in stage III and IV, detection of IL-4, IL-6, and IL-10 mRNA expression were significantly more positive than that in stage I and II.

Our study found that the optimal cutoff value for IL-2, IL- 8, TNF- $\alpha$, PCT, and CRP were $838 \mathrm{U} / \mathrm{mL}$, $46.15 \mathrm{pg} / \mathrm{mL}, 11.95 \mathrm{pg} / \mathrm{mL}, 0.77 \mathrm{pg} / \mathrm{mL}$, and $109.38 \mathrm{mg} / \mathrm{L}$ in GI cancers. However, as the cytokine arrays are multiplex, the prognostic value in different diseases is inconsistent. In our previous study, the cutoff values for IL-2, IL-6, IL-8, and TNF- $\alpha$ were $930.5 \mathrm{U} / \mathrm{mL}$, $50.95 \mathrm{pg} / \mathrm{mL}, 44.1 \mathrm{pg} / \mathrm{mL}$, and $11.95 \mathrm{pg} / \mathrm{mL}$ in critical patients after major surgery (26). Rettig et al. (27) reported the optimal cutoff point of IL-6 was $432 \mathrm{pg} / \mathrm{mL}$ on the first day after surgery. Therefore, our results should be validated in other cancer populations and supported by further studies to examine the prognostic value of these cytokines. In the future, the optimal combination of biomarkers as a prognostic tool should be built and validated in cancer patients.

Although we detected the prognostic value of cytokines in GI cancers, there were still several limitations in our study. First, we analyzed 5 cytokines respectively, although combining these variables may provide more promising information. A prognostic model including multiple cytokine assays should be built to detect the clinical outcomes in cancer or other diseases. Second, we only analyzed the first detection level after SICU admission, sequential and dynamic assessments during SICU stay may provide further evidence for the prediction value of the cytokines in critical GI cancer.

\section{Conclusions}

The current investigation provides a promising insight for 
the correlation between IL-2, IL-8, TNF- $\alpha$, PCT, CRP, and clinical outcomes in critical GI cancers. Prognostic value analyses and identifying potential biomarkers are important in investigating the mechanisms of cancer. Detection of biomarkers with predictive abilities can help clinicians to monitor cancer progression and engender individualize treatment regimens.

\section{Acknowledgments}

The authors sincerely thank Jian Gao for his guidance in data and statistical analysis.

Funding: This work was supported by the Youth Program of Zhongshan Hospital, Fudan University (2019ZSQN01), Fuxing Nursing Program of Fudan University (FNF201945; FNF202007).

\section{Footnote}

Reporting Checklist: The authors have completed the REMARK reporting checklist. Available at https://dx.doi. org/10.21037/jgo-21-334

Data Sharing Statement: Available at https://dx.doi. org/10.21037/jgo-21-334

Conflicts of Interest: All authors have completed the ICMJE uniform disclosure form (available at https://dx.doi. org/10.21037/jgo-21-334). The authors have no conflicts of interest to declare.

Ethical Statement: The authors are accountable for all aspects of the work in ensuring that questions related to the accuracy or integrity of any part of the work are appropriately investigated and resolved. This study was carried out retrospectively. Before the analysis, all participant data were anonymized and deidentified. All procedures performed in this study involving human participants were in accordance with the Declaration of Helsinki (as revised in 2013), and approval to conduct this study was granted by the Ethics Committee on Human Experimentation of Zhongshan Hospital, Fudan University (B2020-107R). Patients provided written informed consent.

Open Access Statement: This is an Open Access article distributed in accordance with the Creative Commons Attribution-NonCommercial-NoDerivs 4.0 International License (CC BY-NC-ND 4.0), which permits the non- commercial replication and distribution of the article with the strict proviso that no changes or edits are made and the original work is properly cited (including links to both the formal publication through the relevant DOI and the license). See: https://creativecommons.org/licenses/by-nc-nd/4.0/.

\section{References}

1. Bray F, Ferlay J, Soerjomataram I, et al. Global cancer statistics 2018: GLOBOCAN estimates of incidence and mortality worldwide for 36 cancers in 185 countries. CA Cancer J Clin 2018;68:394-424.

2. Siegel RL, Miller KD, Jemal A. Cancer statistics, 2018. CA Cancer J Clin 2018;68:7-30.

3. Nair VS, Maeda LS, Ioannidis JP. Clinical outcome prediction by microRNAs in human cancer: a systematic review. J Natl Cancer Inst 2012;104:528-40.

4. Cabrero-de Las Heras S, Martínez-Balibrea E. CXC family of chemokines as prognostic or predictive biomarkers and possible drug targets in colorectal cancer. World J Gastroenterol 2018;24:4738-49.

5. Conlon KC, Miljkovic MD, Waldmann TA. Cytokines in the Treatment of Cancer. J Interferon Cytokine Res 2019;39:6-21.

6. Bagheri V, Memar B, Momtazi AA, et al. Cytokine networks and their association with Helicobacter pylori infection in gastric carcinoma. J Cell Physiol 2018;233:2791-803.

7. Li J, Xu L, Run ZC, et al. Multiple cytokine profiling in serum for early detection of gastric cancer. World J Gastroenterol 2018;24:2269-78.

8. Khosravi N, Stoner L, Farajivafa V, et al. Exercise training, circulating cytokine levels and immune function in cancer survivors: A meta-analysis. Brain Behav Immun 2019;81:92-104.

9. Taniguchi K, Karin M. IL-6 and related cytokines as the critical lynchpins between inflammation and cancer. Semin Immunol 2014;26:54-74.

10. Eldesoky A, Shouma A, Mosaad Y, et al. Clinical relevance of serum vascular endothelial growth factor and interleukin-6 in patients with colorectal cancer. Saudi J Gastroenterol 2011;17:170-3.

11. Fazzari J, Sidhu J, Motkur S, et al. Applying Serum Cytokine Levels to Predict Pain Severity in Cancer Patients. J Pain Res 2020;13:313-21.

12. Panis C, Pavanelli WR. Cytokines as Mediators of PainRelated Process in Breast Cancer. Mediators Inflamm 2015;2015:129034. 
13. Li MX, Liu XM, Zhang XF, et al. Prognostic role of neutrophil-to-lymphocyte ratio in colorectal cancer: a systematic review and meta-analysis. Int J Cancer 2014;134:2403-13.

14. Guthrie GJ, Roxburgh CS, Horgan PG, et al. Does interleukin-6 link explain the link between tumour necrosis, local and systemic inflammatory responses and outcome in patients with colorectal cancer? Cancer Treat Rev 2013;39:89-96.

15. Gunawardene A, Dennett E, Larsen P. Prognostic value of multiple cytokine analysis in colorectal cancer: a systematic review. J Gastrointest Oncol 2019;10:134-43.

16. Qeadan F, Bansal P, Hanson JA, et al. The MK2 pathway is linked to G-CSF, cytokine production and metastasis in gastric cancer: a novel intercorrelation analysis approach. J Transl Med 2020;18:137.

17. Chen ZY, Raghav K, Lieu CH, et al. Cytokine profile and prognostic significance of high neutrophil-lymphocyte ratio in colorectal cancer. Br J Cancer 2015;112:1088-97.

18. Lippitz BE. Cytokine patterns in patients with cancer: a systematic review. Lancet Oncol 2013;14:e218-28.

19. Callegaro D, Miceli R, Mariani L, et al. Soft tissue sarcoma nomograms and their incorporation into practice. Cancer 2017;123:2802-20.

20. Strand KJ, Khalak H, Strovel JW, et al. Expression biomarkers for clinical efficacy and outcome prediction in cancer. Pharmacogenomics 2006;7:105-15.

21. Belluco C, Nitti D, Frantz M, et al. Interleukin-6 blood

Cite this article as: Yan Y, Yu Z, Lu J, Jin P, Tang Z, Hu Y. Predictive values profiling of interleukin-2, interleukin-8, tumor necrosis factor- $\alpha$, procalcitonin, and C-reactive protein in critical gastrointestinal cancer patients. J Gastrointest Oncol 2021;12(4):1398-1406. doi: 10.21037/jgo-21-334 level is associated with circulating carcinoembryonic antigen and prognosis in patients with colorectal cancer. Ann Surg Oncol 2000;7:133-8.

22. Ikeguchi $M$, Hatada T, Yamamoto $M$, et al. Serum interleukin-6 and -10 levels in patients with gastric cancer. Gastric Cancer 2009; 12:95-100.

23. Necula LG, Chivu-Economescu M, Stanciulescu EL, et al. IL-6 and IL-11 as markers for tumor aggressiveness and prognosis in gastric adenocarcinoma patients without mutations in Gp130 subunits. J Gastrointestin Liver Dis 2012;21:23-9.

24. Birgisson H, Tsimogiannis K, Freyhult E, et al. Plasma Protein Profiling Reveal Osteoprotegerin as a Marker of Prognostic Impact for Colorectal Cancer. Transl Oncol 2018;11:1034-43.

25. Liang J, Li Y, Liu X, et al. Relationship between cytokine levels and clinical classification of gastric cancer. Asian Pac J Cancer Prev 2011;12:1803-6.

26. Yan Y, Hu Y, Wang X, et al. The predictive prognostic values of serum interleukin-2, interleukin-6, interleukin-8, tumor necrosis factor- $\alpha$, and procalcitonin in surgical intensive care unit patients. Ann Transl Med 2021;9:56.

27. Rettig TC, Verwijmeren L, Dijkstra IM, et al. Postoperative Interleukin-6 Level and Early Detection of Complications After Elective Major Abdominal Surgery. Ann Surg 2016;263:1207-12.

(English Language Editor: J. Jones) 